\title{
Sustained-release multiple-component cellulose acetate nano-fibers fabricated using a modified coaxial electrospinning process
}

\author{
Jie Yan, Kenneth White, Deng-Guang Yu, Xu-Yao Zhao
}

J.Yan

Research Center for Analysis and Measurement, Donghua University, 2999 North Renmin Road, Songjiang District, Shanghai, 201620 _People's Republic of China

K . W h i t e School of Human Sciences, Faculty of Life Sciences and Computing, London Metropolitan University, 166-220 Holloway Road, London, N7 8DB UK

D.-G. Yu, X.-Y. Zhao

School of Material Science \& Engineering, University of Shanghai for Science and Technology, Shanghai, 200093 China

\begin{abstract}
Two drawbacks of the traditional electrospinning process when used for producing nanofibers for drug release are that clogging of the spinneret is often experienced, and the fibers produced often exhibit a tailing-off of drug release over sustained periods. The present study investigates the preparation of ferulic acid (FA) sustained-release cellulose acetate (CA) nanofibers, in which a third component, polyvinylpyrrolidone (PVP), was included into the nanocomposites for an improved sustained drug release profile. A modified coaxial electrospinning process, in which only organic solvent N,N-dimethylacetamide was used as a sheath fluid, was exploited for a smooth and continuous fabrication of multiple-component nanofibers. Under an applied voltage of $16 \mathrm{kV}$ and an optimized sheath-to-core flow rate ratio of 0.11 , three types of FA/ PVP/CA composite nanofibers (with varied of PVP content) were generated. These nanofibers had higher quality in terms of size and distribution of nanofiber diameter, as indicated by FESEM images. Analysis of double- and triple- omponent nanofibers by XRD, DSC, and ATR-FTIR confirmed the compatibility of components producing homogenous fibers in both cases, but the triple-component nanofibers exhibited better release profiles over sustained periods than the double-component nanofibers in terms of release completeness, reduced_tailing-off, and adjustable release rates. The modified coaxial process and the resulting_multiple-component nanocomposites should provide a new way for developing novel drug sustained materials and drug delivery systems.
\end{abstract}

Keywords: Coaxial electrospinning,_Sustained_drug release, Multiple component nanofibers, Nanocomposites, Tailing off release

\section{Introduction}

Electrospun nanofibers, randomly assembled in the form of a non-woven mat, have been broadly investigated as potential drug delivery systems (DDS), either directly or following further treatment [1-4]. These drug-loaded nanofibers have been increasingly tested in animals with a view to further assessment in clinical tests and for commercialization [5-8]. However, there are two main concerns that remain to be resolved in order for electrospinning to be scaled up from laboratory to industrial applications. One is the mass production of nanofibers, which has been reported in several modified electrospinning processes, such as multiple needle electrospinning, needleless electrospinning, and edge electrospinning $[9,10]$. The other problem is the frequent clogging of the spinneret during the electrospinning process, particularly when a volatile solvent is used for the preparation of spinning solutions $[11,12]$. By replacing traditional single-fluid electrospinning, which creates a fluid-atmosphere interface, with a modified coaxial electrospinning, in which the electrospinnable fluid partially has a 
fluid-liquid-atmosphere interface, clogging of the spinneret is effectively reduced, thereby creating new possibilities for generating highquality nanofibers and preparing fibers from otherwise unspinnable, concentrated polymer solutions [13, 14].

Electrospun nanofiber-based DDS have been developed to provide almost all the types of controlled release profiles of drugs, such as sustained release, immediate release, pulsatile release, delayed release, targeted release, and biphasic or multiple-phase releases [15-18]. The most common way for generating drug-loaded nanocomposites is_by first preparing a co-dissolving solution of a guest drug and a host polymer, which is then followed by the electrospinning process, which removes the solvents and causes the drug to be encapsulated in the solid nanofibers [19,20]. Based on the properties of the filament-forming polymer matrix and by taking advantage of the properties of the non-woven mats such as high surface area, high porosity, and continuous web structure, immediate release of drug can be achieved from PVP and PEO electrospun nanofibers [21], sustained release can be achieved from a variety of pharmaceutical polymers such as ethyl cellulose, cellulose acetate (CA), and chitosan [9, 21], and also man-made polymers such as polyacrylonitrile can be used for possible transdermal or topical applications [22]. Often the drug distributes in the polymer nanofibers in a molecular way because of the favorable secondary interactions between the drug and the polymer matrix, and also because of the fast drying process of electrospinning, causing the physical state of the components in the spinning solutions to be propagated into the solid nanofibers, to generate solid dispersions at a molecular scale without discernable nanoparticles resulting from phase separation [23].

The highly even distribution of drug in the polymer matrix, on the one hand, does favor a sustained drug release profile through a diffusion mechanism when an insoluble, or a degradable, polymer is exploited. On the other hand, this distribution inevitably brings out two negative influences on the sustained-release profiles. One is the initial burst effect, caused by the even, molecular distribution of drug on the nanofibers' surface and by the large surface area of nanofibers [3, 24]. To mitigate, or even eliminate, the initial burst effects, post-treatments of the electrospun nanofiber mats and encapsulations of the drug in the core part of core/sheath nanofibers have been reported, using coaxial electrospinning [25-27]. Another negative influence is that the encapsulated drugs often could not be completely released from the insoluble polymers (sometime only $70 \%$ of the incorporated drug can be released) and/or there may be a long phase of a "tailing-off"of drug release at the end [28]. Sustained drug release has gained considerable attention as a method of administering and maintaining desired drug concentrations in the blood within a specified therapeutic window, or in target tissues within a desired duration of drug delivery [29, 30]. The first study on the application of electrospun nanofibers in pharmaceutics focused on the sustained release of tetracycline hydrochloride using poly(lactic acid) and poly(ethylene-co-vinyl acetate), as well as their blend as filament-forming polymeric matrices [31]. The drug released from the "leveling-off"time period does not effectively sustain drug concentrations at desired levels in the blood and should be eliminated [32]. Different methods, such as control of the tablets' shape, have been tried, to address this problem in traditional DDS [33].

Building on the developments discussed thus far, this study investigates the preparation of drug-loaded CA nanofibers with an additional component, polyvinylpyrrolidone (PVP), for facilitating the complete exhaustion of drug and diminishing the "tailingoff time period, as a means of improving the profile of sustained drug release. A modified coaxial electrospinning process was used to generate the multiple-component nanofibers and the key parameters of the modified coaxial processes (sheath-to-core flow rate ratios and the applied high voltage) were investigated. CA, the acetate ester of cellulose, has been widely investigated for a variety of potential applications in the form of electrospun nanofiber mats, because of its advantageous properties, such as good biocompatibility, biodegradability, regenerative properties, and high affinity with other substances, and hence was used as the filament-forming polymer matrix [26, 34]. Ferulic acid (FA, 4-hydroxy-3-methoxycinnamic acid), an antioxidant capable of scavenging free radicals, was used as the model active ingredient [35, 36]. PVP has a wide variety of applications in medicine, pharmaceutics, and cosmetics, and has been widely used as a template for preparing functional nanofibers for applications such as drug-containing nanofibers, inorganic-organic composites, inorganic nanofibers, precursors for organic peroxide nanofibers, and liposomes. In drug delivery, PVP is widely used as a binder in tableting (wet granulation, dry granulation, direct compression, and effervescent tablets), in film coatings and adhesive gels, and is particularly used as an excipient to prepare solid dispersions for improving the dissolution rates of poorly water-soluble drugs [37]. 
Materials and methods

Materials

CA (white powder; $\mathrm{M}_{\mathrm{w}}=100,000 \mathrm{Da}$, average degree of substitution $=2.45$ ) was purchased from Acros (NJ, USA) and used as received. FA was purchased from Shanghai Winherb Medical Sci \& Tech Development Co., Ltd. (Shanghai, China). PVP K30 $\left(\mathrm{M}_{\mathrm{w}}=58,000\right)$ was obtained from Shanghai Yunhong Pharmaceutical Aids and Technology Co., Ltd. (Shanghai, China). Methylene blue, N,N-dimethylacetamide (DMAc), acetoneacetone, and anhydrous ethanol were purchased from Sinopharm Chemical Reagent Co., Ltd. (Shanghai, China). All reagents were analytical grade and used without further purification. Water was double distilled just before use.

\section{Modified coaxial electrospinning}

The three types of core electrospinnable CA solutions were prepared by first dissolving $10.0 \mathrm{~g}$ of CA and $1.0 \mathrm{~g}$ of FA in $100 \mathrm{~mL}$ of a solvent mixture of acetone:ethanol:DMAc with a volume ratio of 4:1:1. Then 0, 1.0, and $2.0 \mathrm{~g}$ of PVP K30 was co-dissolved in the mixtures. $5 \mathrm{ppm}$ of methylene blue was added to some of the second solutions, containing $1.0 \mathrm{~g}(8.3 \% \mathrm{w} / \mathrm{w}) \mathrm{PVP}$, to enhance visibility for digital observation and optimization of sheath-to-core flow rate ratio. The sheath fluid was pure DMAc.

Two syringe pumps (KDS100 and KDS200, Cole-Parmer, IL, USA), a high-voltage power supply (ZGF $60 \mathrm{kV} / 2 \mathrm{~mA}$, Shanghai Sute Corp., Shanghai, China), and a homemade concentric spinneret were used to conduct the electrospinning process. The homemade concentric spinneret was fabricated simply by inserting a stainless-steel capillary into a T-branch pipe and then welding them together, more details can be found in literature [14]. Following some optimizations, the applied voltage was fixed at $16 \mathrm{kV}$ and the fibers were collected on an aluminum foil at a distance of $20 \mathrm{~cm}$. All electrospinning processes were carried out under ambient conditions $\left(22 \pm 2{ }^{\circ} \mathrm{C}\right.$; relative humidity, $61 \% \pm 4 \%$ ). The electrospinning process was recorded using a digital video recorder (PowerShot A490, Canon, Tokyo, Japan). Since DMAc can be easily retained in the electrospun fiber [38], the electrospun nanofibers were further dried for two days at $40{ }^{\circ} \mathrm{C}$ under vacuum $(320 \mathrm{~Pa})$ in a DZF-6050 Electric Vacuum Drying Oven (Shanghai Laboratory Instrument Work Co. Ltd., China) to facilitate the removal of residual organic solvents and moisture [39]. The other parameters are listed in Table 1.

\section{Characterization}

Morophology

The morphology of the nanofiber mats was assessed using a Quanta FEG450 scanning electron microscope (SEM) (FEI Corporation,Corporation, The Netherlands). Prior to the examination, the samples were rendered electrically conductive by gold sputter coating under a nitrogen atmosphere. The average fiber diameter was determined by measuring diameters in the SEM images at more than 100 different locations using Image J software (National Institutes of Health, MD, USA). The topography of the FA particles was observed under cross-polarized light using an XP-700 polarized optical microscope (Shanghai Changfang Optical Instrument Co., Ltd).

Physical status of components and compatibility

X-ray diffraction (XRD) patterns were obtained on a D/Max-BR diffractometer (RigaKu, Tokyo, Japan) with CuKa radiation within the 20 range of $5^{\circ}-60^{\circ}$ at $40 \mathrm{mV}$ and $30 \mathrm{~mA}$. Differential scanning calorimetry (DSC) was carried out using a MDSC 2910

Table 1 Parameters of the electrospinning processes and their products

\begin{tabular}{|c|c|c|c|c|c|c|c|}
\hline \multirow[t]{2}{*}{ No. } & \multirow[t]{2}{*}{ Electrospinning } & \multirow[t]{2}{*}{ PVP content $(\%)$} & \multicolumn{3}{|c|}{ Flow rate $\left(\mathrm{mL} \mathrm{h}^{-1}\right)$ and ratio } & \multirow[t]{2}{*}{ Morphology $\mathrm{y}^{\mathrm{b}}$} & \multirow[t]{2}{*}{ Diameter (nm) } \\
\hline & & & Sheath ${ }^{\mathrm{a}}$ & Core & Ratio & & \\
\hline $\mathrm{F} 1$ & Single & 8.3 & 0.0 & 1.0 & 0 & Linear & $740 \pm 170$ \\
\hline $\mathrm{F} 2$ & Coaxial & 8.3 & 0.1 & 0.9 & 0.11 & Linear & $570 \pm 80$ \\
\hline F3 & Coaxial & 8.3 & 0.2 & 0.8 & 0.25 & Beads-on-a-string & $250 \pm 130$ \\
\hline $\mathrm{F} 4$ & Coaxial & 0 & 0.1 & 0.9 & 0.11 & Linear & $510 \pm 80$ \\
\hline F5 & Coaxial & 15.4 & 0.1 & 0.9 & 0.11 & Linear & $600 \pm 90$ \\
\hline
\end{tabular}

aSheath fluid was DMAcb In this column, "linear"morphology refers to nanofibers with few beads or spindles on them 
differential scanning calorimeter (TA Instruments Co., DE, USA). Sealed samples were heated at $10^{\circ} \mathrm{C} \cdot \mathrm{min}^{-1}$ from $20^{\circ} \mathrm{C}$ to 250 ${ }^{\circ} \mathrm{C}$ under a nitrogen flow of $40 \mathrm{~mL} / \mathrm{min}$. Attenuated total reflectance (ATR-) Fourier transform infrared (FTIR) analyses were performed on a NicoletNexus 670 FTIR spectrometer (Nicolet Instrument Corporation, WI, USA) from $500 \mathrm{~cm}^{-1}$ to $4000 \mathrm{~cm}^{-1}$ at a resolution of $2 \mathrm{~cm}^{-1}$.

Hydrophilicity of nanofibers and in vitro dissolution tests

A DSA100 drop shape analysis instrument (Kru" ss GmbH, Hamburg, Germany) was used to explore the interfacial tensions between the polymer solutions and nozzles of the spinnerets.

In vitro dissolution tests were carried out according to the Chinese Pharmacopoeia (2005 ed.) Method II, a paddle method in which an RCZ-8A dissolution apparatus (Tianjin University Radio Factory, Tianjin, China) was used. About 200 mg of drugloaded nanofibers were placed in $900 \mathrm{~mL}$ of physiological saline (PS; $0.9 \mathrm{wt} \%$ ) at $37 \pm 1{ }^{\circ} \mathrm{C}$. The instrument was then set to 50 $\mathrm{rpm}$, providing sink conditions in which $\mathrm{C}<0.2 \mathrm{C}_{\mathrm{s}}$. At predetermined time points, $5.0 \mathrm{~mL}$ samples were withdrawn from the dissolution medium and replaced with fresh medium to maintain a constant volume. After filtration through a j.tm0.22-j.tm membrane (Millipore, MA, USA) and appropriate dilution with PS, the samples were analyzed at $\lambda_{\max }=322 \mathrm{~nm}$ using a UV-Vis spectrophotometer (UV-2102PC, Unico Instrument Co. Ltd., Shanghai, China). The cumulative amount of FA released at each time point was reverse calculated from the data obtained against a predetermined calibration curve. Experiments were carried out six times and the results are presented as mean values. Drug-exhausted nanofibers were dried naturally and observed using FESEM.

Results and discussion

The modified coaxial electrospinning and the applied voltage

A schematic diagram of the modified coaxial electrospinning process with solvent as sheath fluid is shown in Fig. 1a. A homemade concentric spinneret was used to carry out the modified process (Fig. 1b). The critical voltage was needed to be applied to a fluid to initiate Taylor cone formation and produce the straight thinning jet $\left(\mathrm{VcV}_{c}\right)$ which has a close relationship with the diameter of the sheath part of the concentric spinneret [40]:

$V_{c} \cdot \frac{\sqrt{\frac{1}{2}}}{R}$

where $V_{c}$ is the critical voltage for a jet emanating from the meniscus tip, $d$ is the electrode separation, is the permittivity, $y$ is the surface tension, and $\mathrm{R}$ is the principal curvature of the liquid meniscus. A small diameter of the spinneret's orifice means a large $\mathrm{R}$, and thus a small $\mathrm{V}_{\mathrm{c}}$ to initiate the coaxial electrospinning process. The homemade spinneret used in the study has an outer and inner diameter of 1.2 and $0.3 \mathrm{~mm}$, respectively (Fig. 1b), facilitating the coaxial electrospinning process. In addition, the inner capillary projects a little from the surface of the outer capillary, which is expected to make the sheath DMAc fully envelop the core co-dissolving spinnable solutions.

Clogging is a critical but common problem experienced during electrospinning, especially when a high-volatility solvent is used to prepare a polymer solution (Yu et al., 2013) (18). When the sheath fluid flow rate was adjusted to $0 \mathrm{~mL} / \mathrm{h}$ to conduct the traditional single fluid electrospinning process, the Taylor cone enlarged quickly until the electrospinning process was totally stopped (Fig. 2a). Thus, manual removal of the semi-solid substance at the nozzle of the spinneret was needed from time to time for continuous preparation of nanofibers. When the modified coaxial electrospinning was carried out to prepare the composite nanofibers, two syringe pumps were used to drive the sheath and core fluids independently (Fig. 2b). An alligator clip was used to connect the inner stainless-steel capillary with the high-voltage supply (Fig. 2c). With DMAc as sheath fluid and under a sheath-to-core flow rate ratio of 0.11 , the arrangement produced a typical fluid jet trajectory, in which a Taylor cone was followed by a straight fluid jet and a bending and whipping instability region (Fig. 2d). As the applied voltage increased from $0 \mathrm{kV}$ to $16 \mathrm{~V}$, the core-shell droplet was quickly transformed from a round shape (Fig. 2 e1-e3) to a cone shape, i.e., the well-known Taylor cone. The compound Taylor cone is clearly composed of two parts with the sheath solvent fully surrounding the core polymer solutions, as indicated by the methylene blue in Fig. 2 e3. When sheath solvent is used to surround the core CA solution during the electrospinning, it can effectively prevent the fast evaporation of the solvent from the surface of the core CA solutions, retarding the formation of a surface "skin", and hence smooth the electrospinning process [4]. 
Morphology of the nanofibers

The influence of sheath fluid flow rate

The sheath-to-core flow rate ratio is one of the most important parameters in the modified coaxial electrospinning process. To determine a suitable value of the sheath flow rate, three sheath-to-core flow rate ratios were used $0,0.11$, and 0.25 , with a fixed total flow rate through the spinneret of $1.0 \mathrm{~mL} / \mathrm{h}$ (Table 1), to generate different types of nanofibers. Their FESEM images are shown in Fig. 3. All three kinds of nanofiber have a linear structure. However, when the electrospinning was carried out with a sheath flow rate of 0 (i.e., as single fluid electrospinning), thicker fibers were produced with a wider diameter distribution and an average diameter of $740 \pm 170 \mathrm{~nm}$ (Fig. 3a). When the sheath-to-core flow rate ratio was increased to 0.25 , the resultant nanofibers had many beads and spindles (Fig. 3c), although the fibers were thinner with an average diameter of $250 \pm 130 \mathrm{~nm}$. In contrast, a more optimal sheath-to-core flow rate ratio of 0.11 resulted in nanofibers with higher quality. The nanofibers F2 have smaller diameters and a narrower distribution of $620 \pm 120 \mathrm{~nm}$ than F1 and a better linear structure than F3 (without any beads/spindles or very thick fibers within them). Based on these results, a sheath-to-core flow rate ratio of 0.11 was adopted in the present study to generate nanofibers with different contents of PVP in the FA-loaded CA nanofibers.

The influence of PVP content on the nanofibers

FESEM images of nanofibers with different contents of PVP are shown in Fig. 4. All three types of nanofibers have fine linear structures with few spindles or beads in them. With the increase of PVP in the nanofibers from F4 (Fig. 4a), to F2 (Fig. 4b) and F5 (Fig. 4c), the average diameter increased slightly from $510 \pm 80$, to $570 \pm 80$ and $600 \pm 90 \mathrm{~nm}$, respectively.

The physical status of the components in the nanofibers

FA, a slight yellow powder to the naked eye, comprised polychrome crystals in the form of prisms or needles, and revealed a rough surface under cross-polarized light (Fig. 5b). The presence of many distinct peaks (Fig. 5a) in the XRD pattern of pure FA similarly verified that FA was present as a crystalline material. The diffraction patterns of raw CA powders exhibit a diffuse background pattern with one diffraction halo, indicating that the polymer is amorphous. The spectrum of amorphous PVP K30 was characterized by the complete absence of any diffraction peak. From Fig. 5a, it is clear that the characteristic diffraction peaks of crystalline FA are completely absent for the three types of composite nanofibers. These results suggest that all the FA in the doublecomponent nanofibers F4 and in the multiple-component composite nanofibers F2 and F5 were amorphous.

The DSC thermograms of individual components and composites in nanofibers are shown in Fig. 6. The DSC curve of pure FA exhibited a single endothermic response corresponding to a melting point of $173.4{ }^{\circ} \mathrm{C} \mathrm{DHf}=-211.7 \mathrm{~J} \mathrm{~g}^{-1}$ ). Being amorphous polymers, PVP and CA powders do not show fusion peaks or obvious phase transitions. DSC thermograms of the double-component FA/CA nanofibers F4 and the triple-component FA/PVP/CA nanofibers did not show any peaks characteristic of FA melting, suggesting that the drug was no longer present as a crystalline material but had been converted into an amorphous state in the composite nanofibers. The results obtained from DSC and XRD confirmed that FA was highly distributed in the nanofibers matrix and was present in an amorphous manner where the original structure of the pure materials had been lost. These results concurred with the observations from FESEM, in which no separate particles could be discerned in the nanofibers.

The molecular structures of the three components are shown in Fig. 7. FA and CA molecules possess free hydroxyl groups (-OH) and these could act as potential proton donors for hydrogen bonding. $\mathrm{CA}, \mathrm{FAFA}$, and PVP molecules have carbonyl groups $(-\mathrm{C}=\mathrm{O})$ and so could act as proton acceptors. Therefore, it can be speculated that hydrogen bonding interactions can occur within the composite nanofibers through interactions between these groups. ATR-FTIR spectra (at the range of 500-2500 $\mathrm{cm}^{-1}$ ) are included in Fig. 7. A different type of hydrogen bonding between FA molecules in the FA crude particles is possible because FA molecules have both $-\mathrm{OH}$ groups and $-\mathrm{C}=\mathrm{O}$ groups. This can be verified by the ATR-FTIR spectra in which sharp peaks were visible for pure crystalline FA at 1689,1663 , and $1619 \mathrm{~cm}^{-1}$, representing the stretching vibration of $-\mathrm{C}=\mathrm{O}$ groups in the different structures of the crystal lattice. However, all the peaks for FA disappeared in the ATR-FTIR spectra of the composite nanofibers F2, F4, and F5. Only one large peak at $1728 \mathrm{~cm}^{-1}$ for composite nanofibers was noted. Meanwhile, numerous peaks at the finger region of the FA spectrum were absent in the spectra of nanofibers F2, F4, and F5. These results jointly suggest that there are fewer interactions between the FA molecules, but extensive hydrogen bond formation between the CA and FA molecules and also between PVP and FA molecules. The hydrogen bonding, combined with the possible electrostatic and hydrophobic interactions, should provide an environment that stabilizes the structure, giving a high degree of compatibility between the components that make up 
the nanofibers [34] .]. This in turn creates a homogeneous structure regardless of whether the mixture is of double components of FA and CA or triple components of FA, CA, and PVP.

The hydrophilicity of nanofibers and the improved sustained-release profiles

$\mathrm{CA}$ is an insoluble and hydrophobic polymer and FA is a poorly water-soluble active pharmaceutical ingredient. In sharp contrast, PVP has super hygroscopic and hydrophilic properties and PVP nanofibers containing a drug which is poorly soluble in water can dissolve within several seconds [15]. To investigate the hydrophilicity of the composite nanofibers produced in this study, water contact angles were determined by using a drop shape analysis instrument to place droplets of 3 j.tL on the fiber mats. As anticipated, the composite nanofiber mats exhibited increasingly smaller contact angles, $94 \pm 15^{\circ}, 77 \pm 11^{\circ}$, and $54 \pm 8^{\circ}$ ( $\mathrm{n}>$ 10), as the content of PVP increased from 0,0 to $8.3 \%$ and to $15.4 \%$ for nanofibers F4, F2, and F5, respectively. Examples of typical images are shown in Fig. 8.

The in vitro release profiles of FA from the composite nanofibers are shown in Fig. 9 and some characteristic release parameters are listed in Table 2. In the first hour, 28.6, 30.4, and $31.2 \%$ of the loaded FA was released from the composite nanofibers F4, F2, and F5, respectively (Fig. 9a1). The initial burst effect of the triple-component composite nanofibers F2 and F5 was slightly larger than that of the double-component nanofibers F4, which should be attributed to the hydrophilic properties and easy dissolution of PVP. Electrospun nanofiber-based DDS often have an initial burst drug release of around 30\% [17, 38] due to the huge surface area of nanofibers, the distribution of drug on their surface, and also the amorphous status of drug. Further improvement of the sustained-release profiles should inhibit the burst release by using blank polymer coating on the surface [22, 26] or fabricating nanofibers with gradient distributions of drug in the core-sheath nanofibers, which is under study in our group.

During the in vitro dissolution processes, PVP could act as the pore-forming agent to form passages for the FA entrapped in the inner part of the CA nanofibers to be gradually released by diffusion, and thus facilitates the fast release and exhaustion process of loaded FA from the CA matrix, and completely avoids the entrapment of FA in insoluble CA. As the PVP content in the

composite nanofibers increases, the time taken to release 50 and $90 \%$ of the contained drug shortens quickly (Table 2). After $9 \mathrm{~d}$ in the in vitro dissolution tests, F4 nanofibers could release $98.6 \%$ of the drug content. The "tailing-off"release time periods can be estimated from the time of $90 \%$ release to the time of $98.6 \%$ release. The time periods are 31.4, 34.6, and 70.4 h for nanofibers F5, F2, and F4, respectively. The drug released in this time period often fails to maintain the desired drug concentrations in the blood within a specified therapeutic window and should be avoided [33]. Regardless of the initial burst release and the final tailing-off release, the drug release in the middle time periods from the nanofibers can be modeled according to zero-order kinetics (Table 2 and Fig. 9 a2). All the three nanofibers showed a time period of linear release. The drug release rate increased and the time period shortened as the PVP content increased in the nanofibers, providing a method for manipulating the drug release rate. FESEM observations of the CA nanofibers F4, F2, and F5 after exhaustion of drug are shown in Fig. 9,b1-b3, respectively. Based on the above discussion, it can be concluded that the triple-component nanofibers F2 and F5 showed better sustained-release profiles than the double-component nanofibers F4 in that the former could release all the active ingredient with a shorter time of futile "tailingoff'release, and the release rate could be manipulated through the content of additional third component to a certain extent.

Table 2 Comparison of the release parameters of CA nanofibers with different contents of PVP

\begin{tabular}{|c|c|c|c|c|c|c|c|c|}
\hline \multirow{2}{*}{$\begin{array}{l}\text { Nanofibe } \\
\text { No. }\end{array}$} & \multirow{2}{*}{$\begin{array}{l}\text { PVP content } \\
(\%)\end{array}$} & \multirow{2}{*}{$\begin{array}{l}\underset{(\%)}{\mathrm{a}} \\
(\% \min \\
\end{array}$} & \multirow{2}{*}{$\begin{array}{l}\mathrm{b} \\
\mathrm{t}+\mathrm{n} \% \\
\text { (h) }\end{array}$} & \multirow{2}{*}{$\begin{array}{l}\text { c } \\
\text { t9o \% } \\
\text { (h) }\end{array}$} & \multirow{2}{*}{$\begin{array}{l}\text { d } \\
\text { trailine } \\
\text { (h) }\end{array}$} & \multicolumn{3}{|c|}{ Linear release property } \\
\hline & & & & & & Equation $^{\mathrm{e}}$ & $\begin{array}{l}\text { Range }^{\mathrm{f}} \\
\text { (h) }\end{array}$ & $\mathrm{Rg}$ \\
\hline F4 & 0 & 28.6 & 28.7 & 145.7 & 31.4 & $\mathrm{Q}_{4}=35.9+0.38 \mathrm{t}$ & $1-168$ & 0.9858 \\
\hline $\mathrm{F} 2$ & 8.3 & 30.4 & 16.8 & 119.3 & 34.6 & $\mathrm{Q}_{2}=38.8+0.44 \mathrm{t}$ & $1-144$ & 0.9773 \\
\hline F5 & 15.4 & 31.2 & 11.7 & 88.6 & 70.4 & $\mathrm{Q}_{5}=39.6+0.61 \mathrm{t}$ & $1-96$ & 0.9764 \\
\hline
\end{tabular}

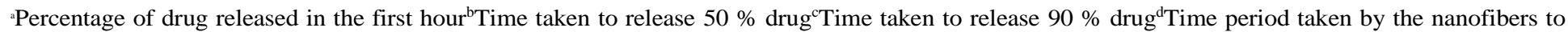

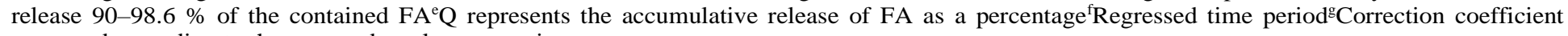
regressed according to the zero-order release equation 
Conclusions

Both double-component FA-loaded CA nanofibers and triple-component FA/PVP-loaded CA nanofibers were fabricated through modified coaxial electrospinning processes. With DMAc as a sheath fluid, the preparation of composite nanofibers was continuous and smooth. Under a condition of a sheath-to-core flow rate ratio of 0.11 and a sheath and core total fluid rate of $1.0 \mathrm{~mL} \mathrm{~h}^{-1}$, FA/PVP-loaded CA nanofibers with different contents of PVP were generated. FESEM observations demonstrated that these nanofibers had higher quality in terms of nanofibers' diameter and distribution. The triple-component nanofibers exhibited better sustained-release profiles than the double-component nanofibers in terms of release completeness, tailing-off release time period, and release rates that could be modulated, even though all the nanofibers were composites, as verified by XRD and DSC tests. The modified coaxial process and the resultant multiple-component nanocompsites should provide a new way for developing novel materials for sustained drug release and transdermal drug delivery.

Acknowledgements This work was supported by the National Science Foundation of China (No. 51373101), the Natural Science Foundation of Shanghai (No. 13ZR1428900), the Key Project of the Shanghai Municipal Education Commission (No. 13ZZ113), the Innovation Project of the University of Shanghai for Science and Technology (No. 3XGM01), and the Innovation Project of the College Student Fund Committee (No. SH201210252153).

\section{References}

1. Li D, Xia Y (2004) Adv Mater 16(14):1151

2. Goh YF, Shakir I, Hussain R (2013) J Mater Sci 48:302748:3027. doi:10.1007/s10853-013-7145-8

3. Liu W, Thomopoulos S, Xia Y (2012) Adv Healthcare Mater 1:10

4. Yan J, Yu DG (2012) J Mater Sci 47:713847:7138. doi:10.1007/s10853-012-6653-2

5. Zahedi P, Rezaeian I, Jafari SHassan (2013) J Mater Sci 48: 314748:3147. doi:10.1007/s13233-013-1064-Z

6. Ravichandran R, Venugopal JR, Sundarrajan S, Mukherjee S, Sridhar R, Ramakrishna S (2012) Int J Cardiol doi:10.1016/ j.ijcard.2012.04.045

7. Thien DVH, Hsiao SW, Ho MH, Li CH, Shih JL (2013) J Mater Sci 48:164048:1640. doi:10.1007/s10853-012-6921-1

8. Nguyen TTT, Ghosh C, Hwang SG, Tran L D, Park JS (2013) J Mater Sci doi :10.1007/s10853-013-7527-y

9. Thoppey NM, Gorga RE, Bochinski JR, Clarke LI (2012) Macromolecules 45:6527

10.Niu H, Lin T (2012) J Nanomater doi:10.1155/2012/725950

11.Kanjanapongkul K, Wongsasulak S, Yoovidhya T (2010) Chem Eng Sci 65:5217

12.Kanjanapongkul K, Wongsasulak S, Yoovidhya T (2010) J Appl Polym Sci 118:1821

13.Yu DG, Branford-White C, Chatterton NP, White K, Zhu LM, Shen XX, Nie W (2010) Macromolecules 43:10743

14.Yu DG, Branford-White C, Bligh SWA, White K, Chatterton NP, Zhu LM (2011) Macromol Rapid Commun 32:744

15.Yu DG, Shen XX, Branford-white C, White K, Bligh SWA, Zhu LM (2009) Nanotechnology doi:10.1088/0957-4484/20/5/ 055104

16.Li G, Chen Y, Cai Z, Li J, Wu X, He X, Zhao Z, Lan P, Li Y (2013) J Mater Sci 48:618648:6186. doi:10.1007/s10853-0137415- 5

17.Huang W, Zou T, Li S, Jing J, Xia X, Liu X (2013) AAPS PharmSciTech 14:675

18.Yu DG, Wang X, Li XY, Chian W, Li Y, Liao YZ (2013) Acta Biomater 9:5665

19.Tian L, Prabhakaran MP, Ding X, Kai D, Ramakrishna S (2012) J Mater Sci 47:3272328147:3272-3281. doi:10.1007/s10853011-6166-4 
20.Tungprapa S, Jangchud I, Supaphol P (2007) Polymer 48:5030

21.Sill TJ, von Recum HA (2008) Biomaterials 29:1989

22.Wu XM, Branford-White C, Yu DG, Chatterton NP, Zhu LM (2011) Colloid Surf B 82:247

23.Li X, Kanjwal MA, Lin L, Chronakis IS (2013) Colloid Surf B 103:182

24.Chunder A, Sarkar S, Yu Y, Zhai L (2007) Colloid Surf B 58:172

25.Yu DG, Li XY, Wang X, Chian W, Liao YZ, Li Y (2013) Cellulose 20:379 27.Yu DG, Chian W, Wang X, Li XY, Li Y, Liao YZ (2013) J Membr Sci 428:150

27.Yu DG, Branford-White C, Li L, Wu XM, Zhu LM (2010) J Appl Polym Sci 117:1509

28.Tseng YY, Liao JY, Chen WA, Kao YC, Lin SJ (2013) Expert Opin Drug Deliv 10:879

29.Xiang A, McHugh AJ (2011) J Membr Sci 371:211

30.Kenawy ER, Bowlin GL, Mansfield K, Layman J, Simpson DG, Sanders EH, Wnek GE (2002) J Control Release 81:57

31.Kim CJ (1999) Eur J Pharm Sci 7:237

32.Kim CJ (1995) Pharm Res 12:1045

33.Tian Y, Wu M, Liu R, Li Y, Wang D, Tan J, Wu R, Huang Y (2011) Carbohydr Polym 83:743

34.Li X, Lin L, Zhu Y, Liu W, Yu T, Ge M (2013) Polym Compos 34:282

35.Anselmi C, Centini M, Maggiore M, Gaggelli N, Andreassi M, Buonocore A, Beretta G, Facino RM (2008) J Pharm Biomed Anal 46:645

36.Bu“ hler V (1998) Kollidon ${ }^{\circledR}$ :Polyvinylpyrollidone for the Pharmaceutical Industry, 2nd edn. BASF Aktiengesellschaft Feinchemie, Ludwigshafen

37.Rodriguez K, Gatenholm P, Renneckar S (2012) Cellulose 19:1583

38.Wu XM, Branford-White C, Zhu LM, Chatterton NP, Yu DG (2010) J Mater Sci Mater Med 21:2403

39. Yeo LY, Friend JR (2006) J Exp Nanosci 2:177 


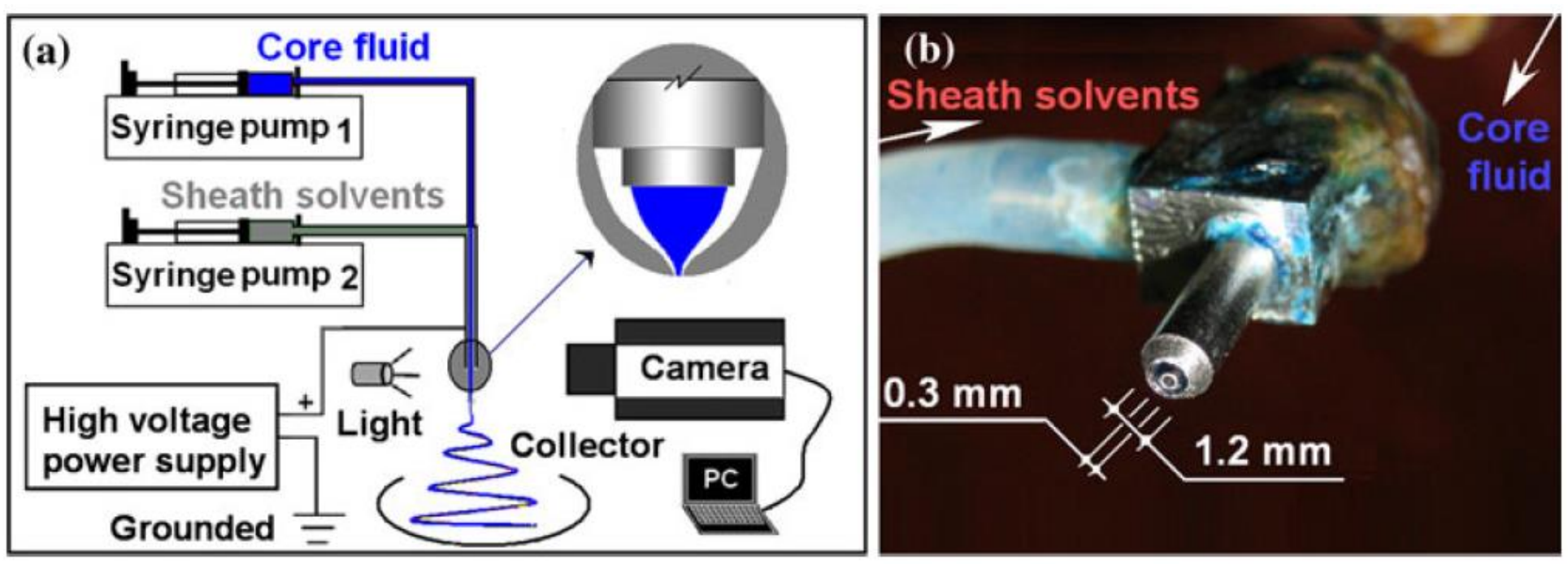

Fig. 1 (a) A diagram of the modified coaxial electrospinning process. (b) A close-up view of the concentric spinneret. 


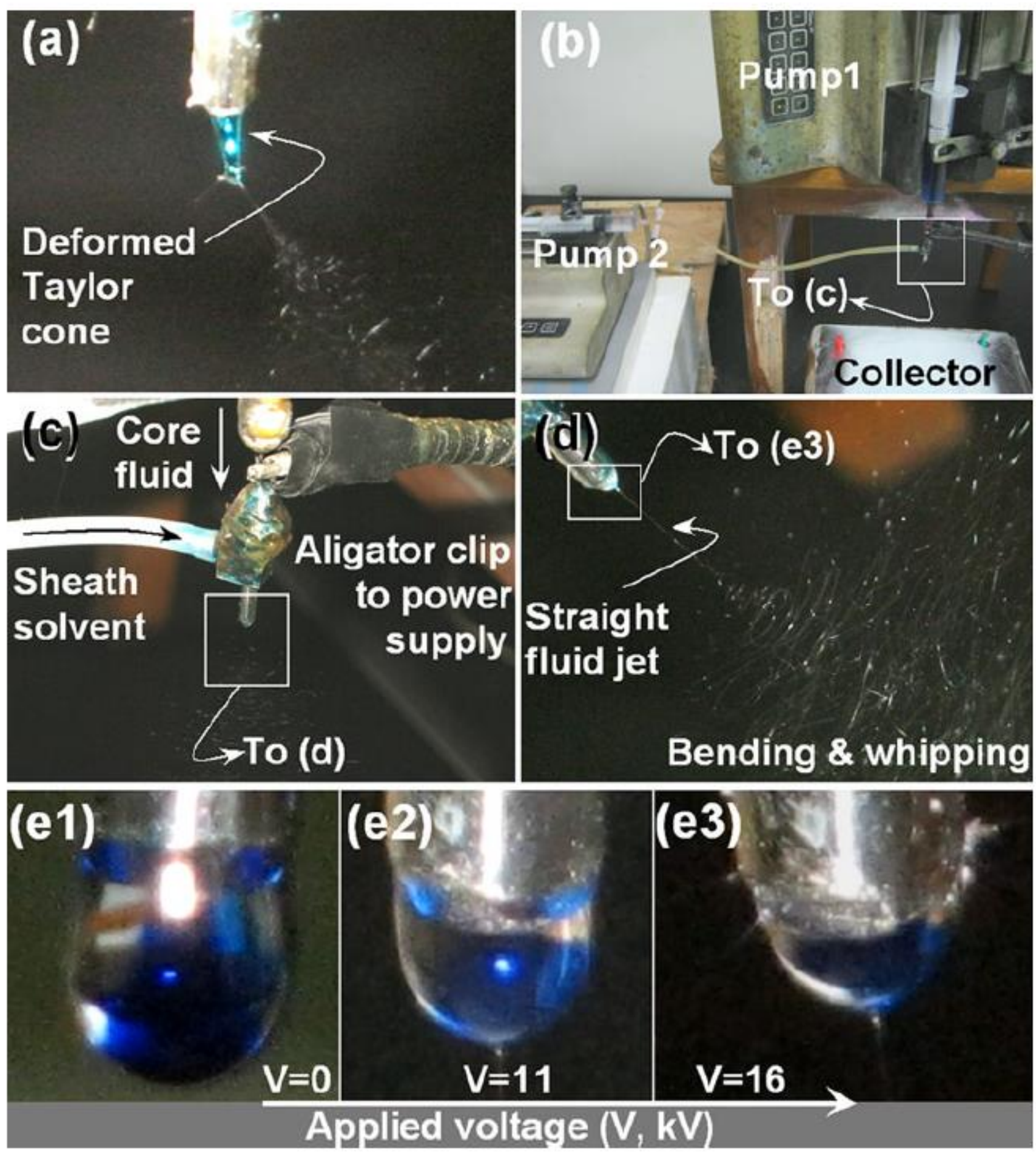

Fig. 2 Observations of the modified coaxial and a single-fluid electrospinning processes: (a) a deformed Taylor cone resulting from the progressive development of clogging in a single-fluid electrospinning; (b) the arrangement of the apparatus for conducting the modified coaxial electrospinning; (c) the connection of the concentric spinneret with the syringe pumps and the power supply; (d) a typical coaxial electrospinning process with DMAc as sheath fluid and under a sheath-to-core flow rate ratio of $0.11 ;(\mathrm{e} 1-\mathrm{e} 3)$ a process of core/shell droplets transforming into a compound Taylor cone as the applied voltage increased from 0 to 11 , and then to $16 \mathrm{kV}$ (taken under a magnification of 12). 

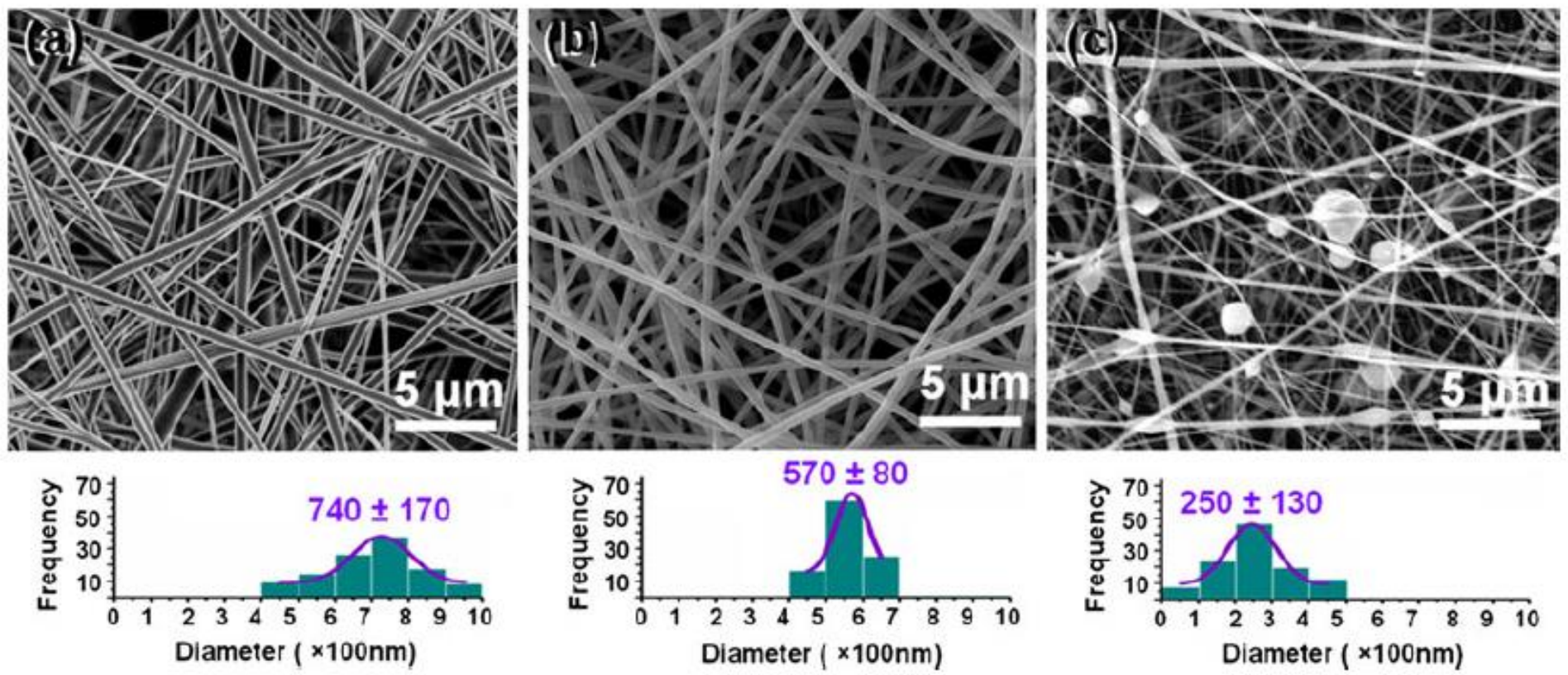

Fig. 3 FESEM images of nanofibers F1 (a), F2 (b), and F3 (c), which were generated under different sheath-to-core flow rate ratios of $0,0.11$, and 0.25 , respectively. The total flow rate of sheath and core fluid was $1.0 \mathrm{~mL} \mathrm{~h}^{-1}$
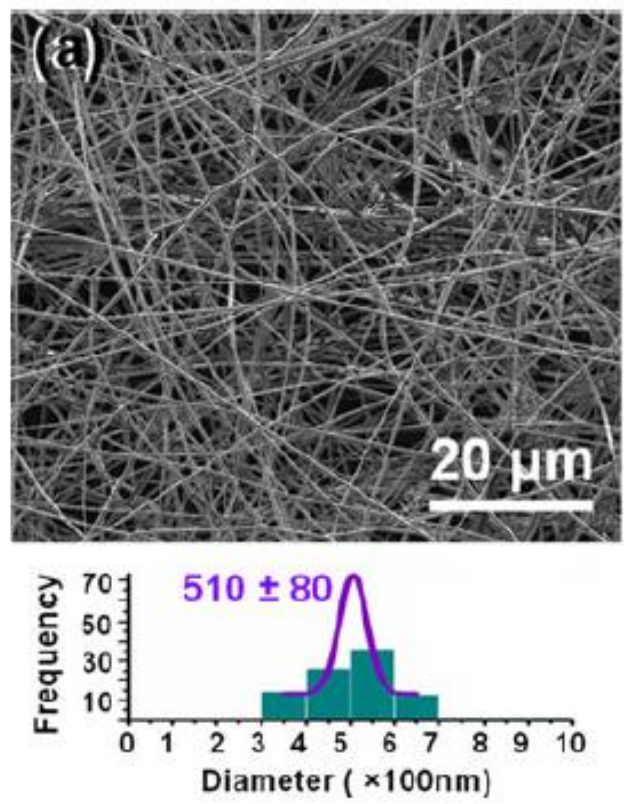
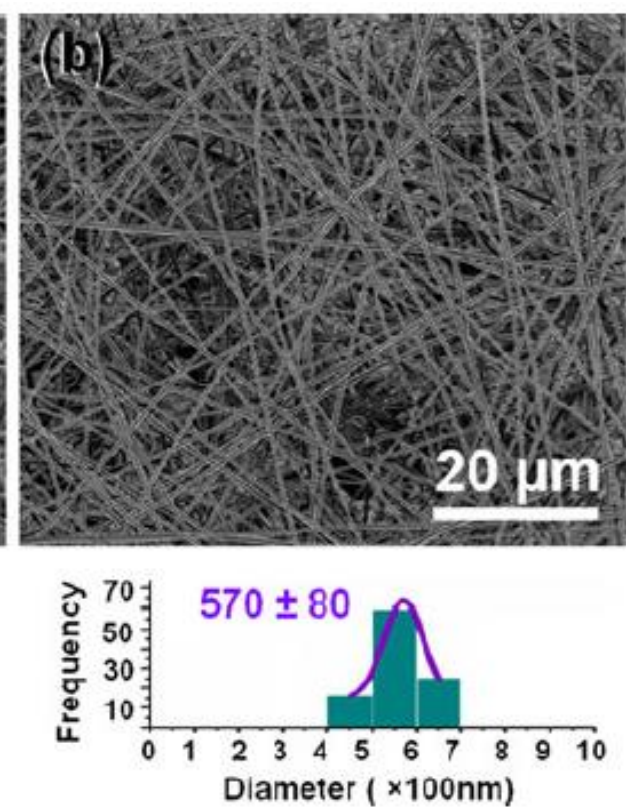
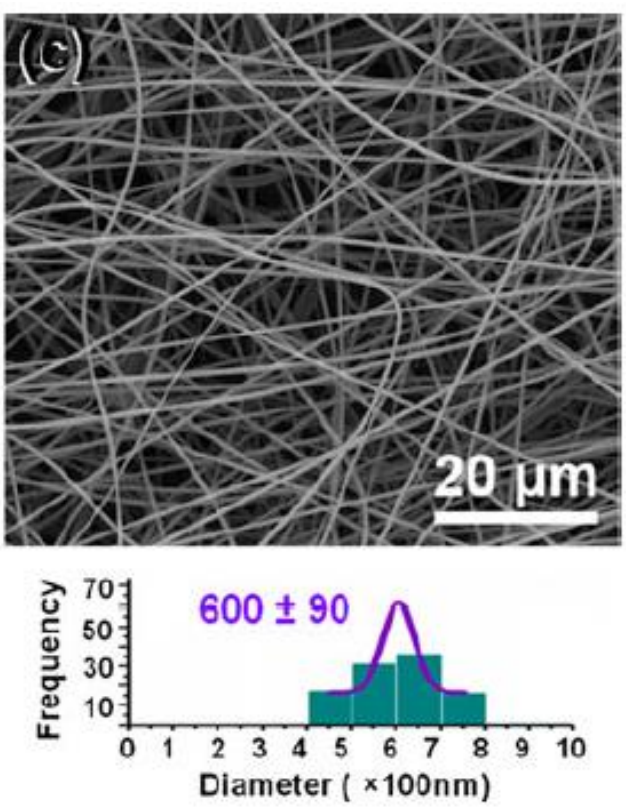

Fig. 4 FESEM images of nanofibers F2 (a), F4 (b), and F5 (c), with PVP contents of 8.3, 0, and $15.4 \%$ $(\mathrm{w} / \mathrm{w})$, respectively, which were generated using a sheath-to-core flow rate ratio of 0.11 and a total flow rate from the spinneret of $1.0 \mathrm{~mL} \mathrm{~h}^{-1}$ 

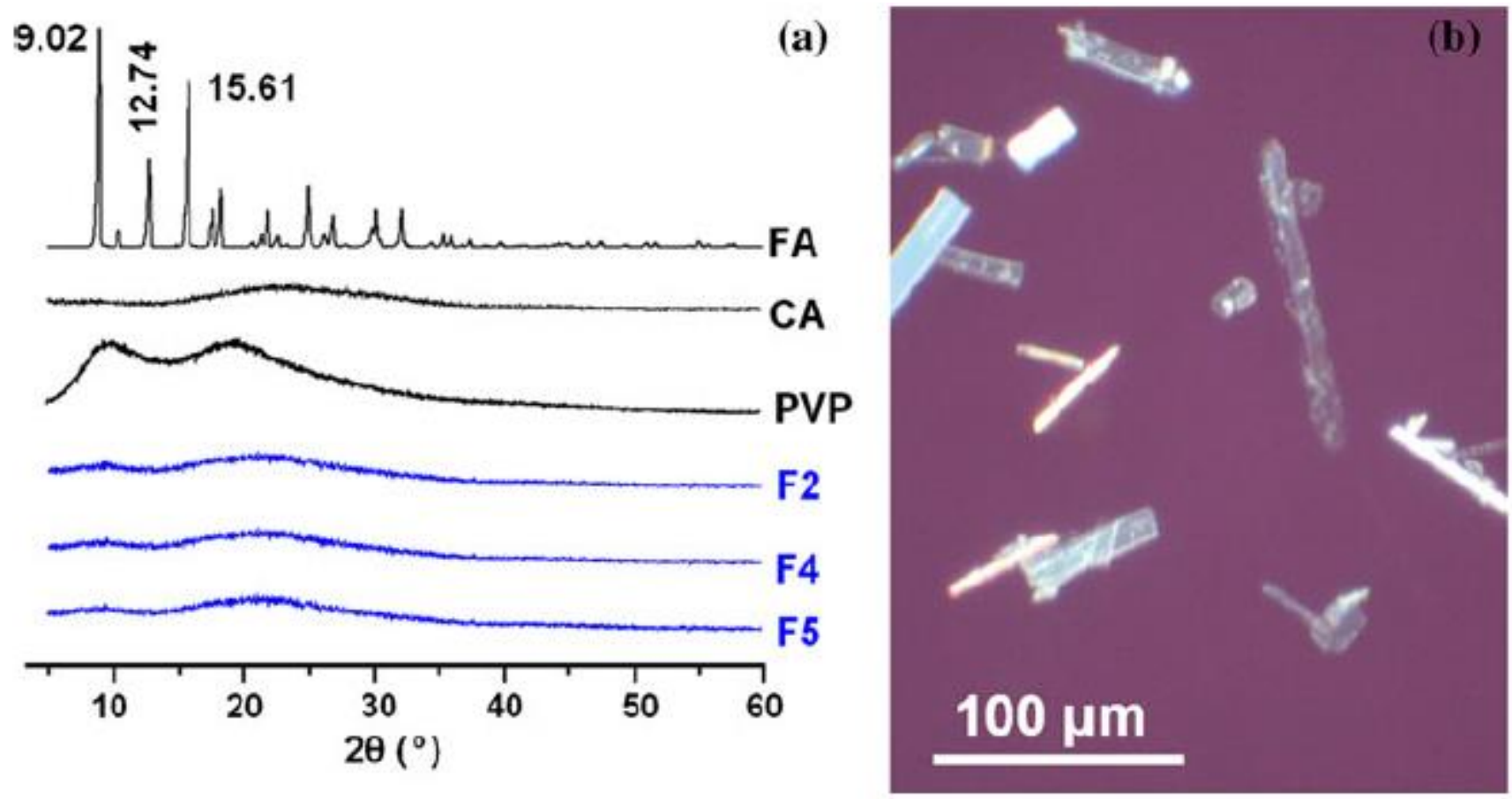

Fig. 5 (a) XRD patterns of the raw materials and their composite nanofibers. (b) Crystals of ferulic acid viewed under cross-polarized light. 


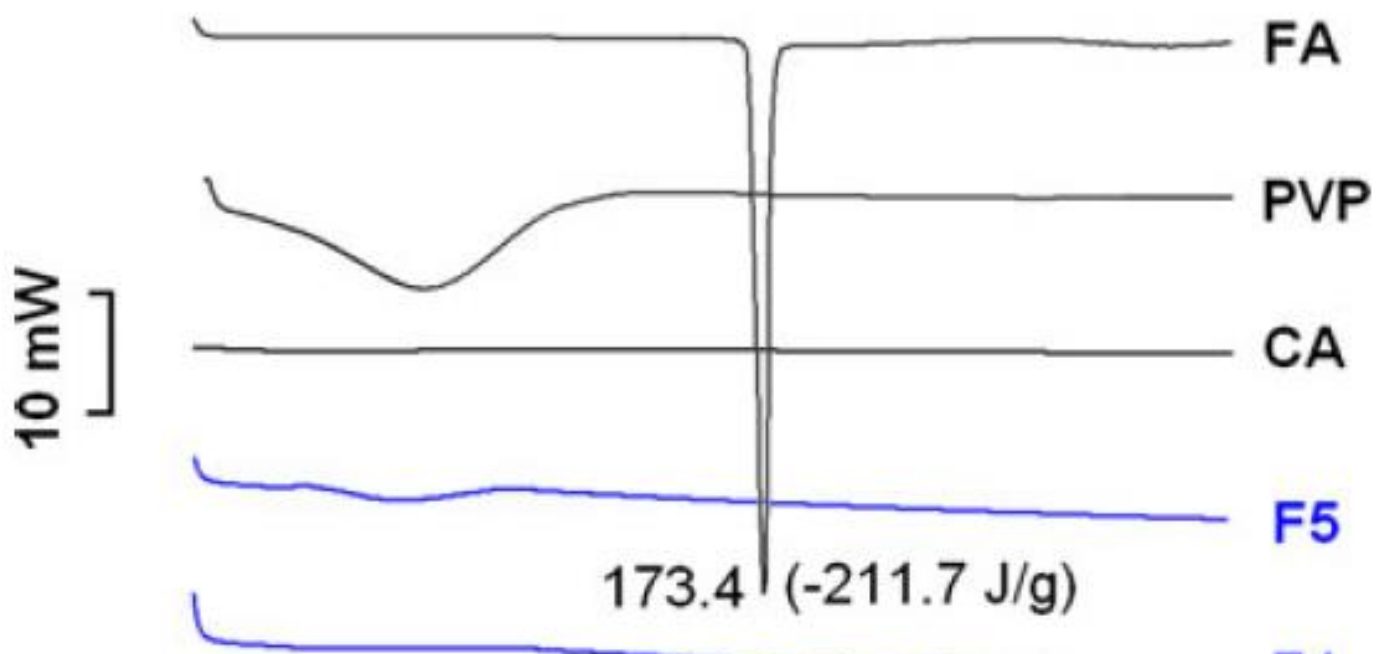

F4

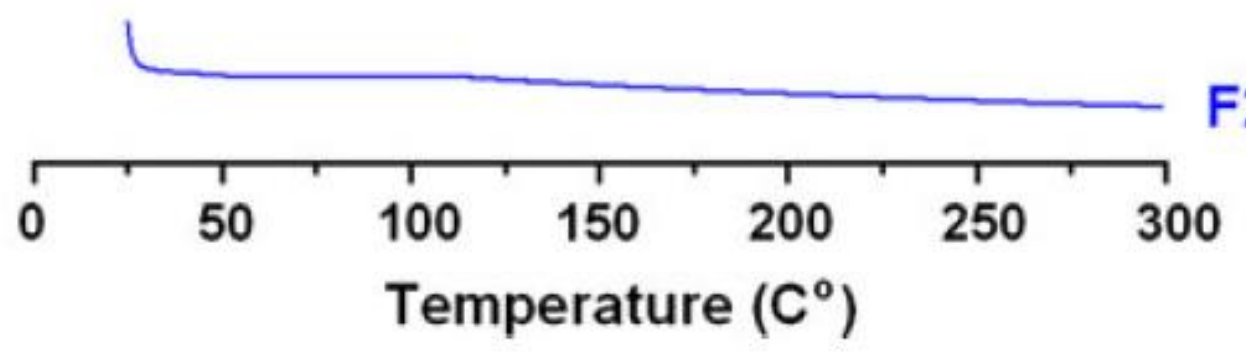

Fig. 6 DSC curves of individual components, a double-component (FA and CA) nanofiber F4, and two triple-component (FA, CA, and PVP) nanofibers F2 and F5 

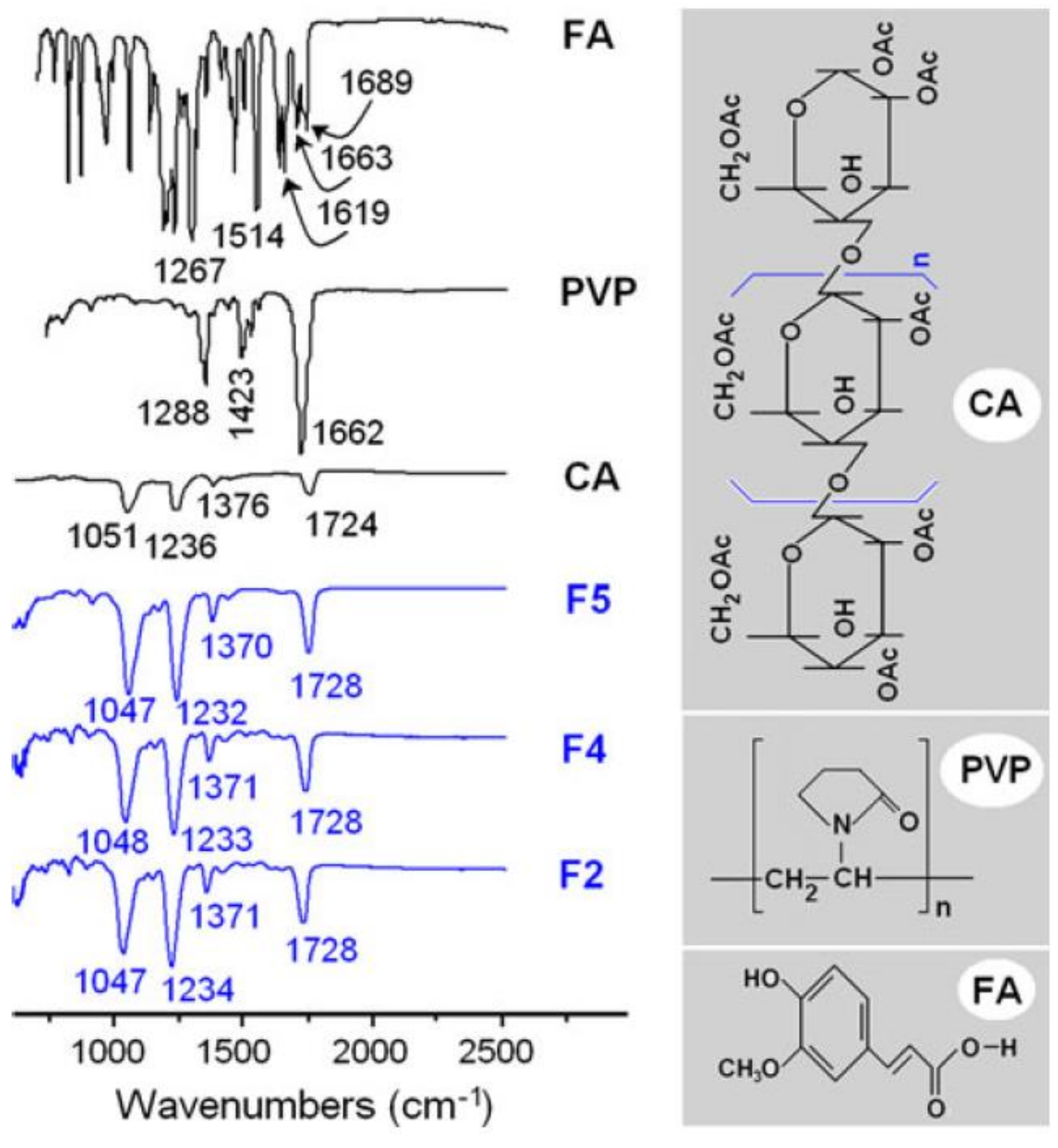

Fig. 7 ATR-FTIR spectra of the raw materials and their composite nanofibers 


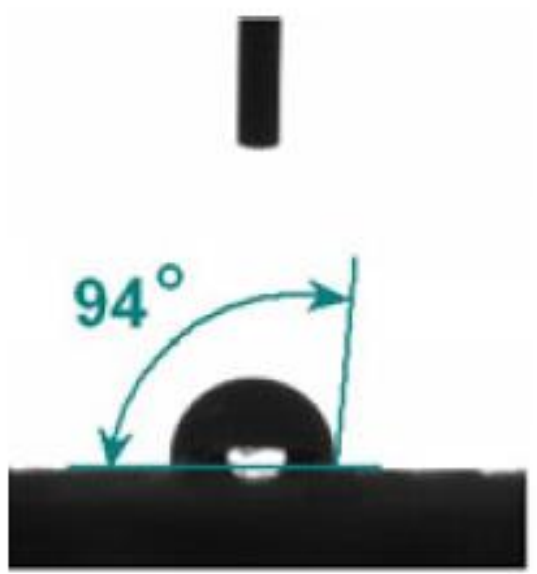

F4

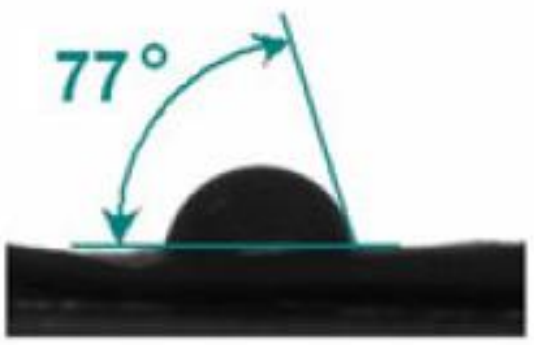

F2

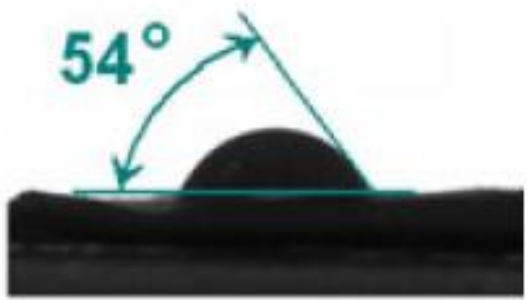

F5

Fig. 8 The water contact angles on the nanofibers F4, F2, and F5
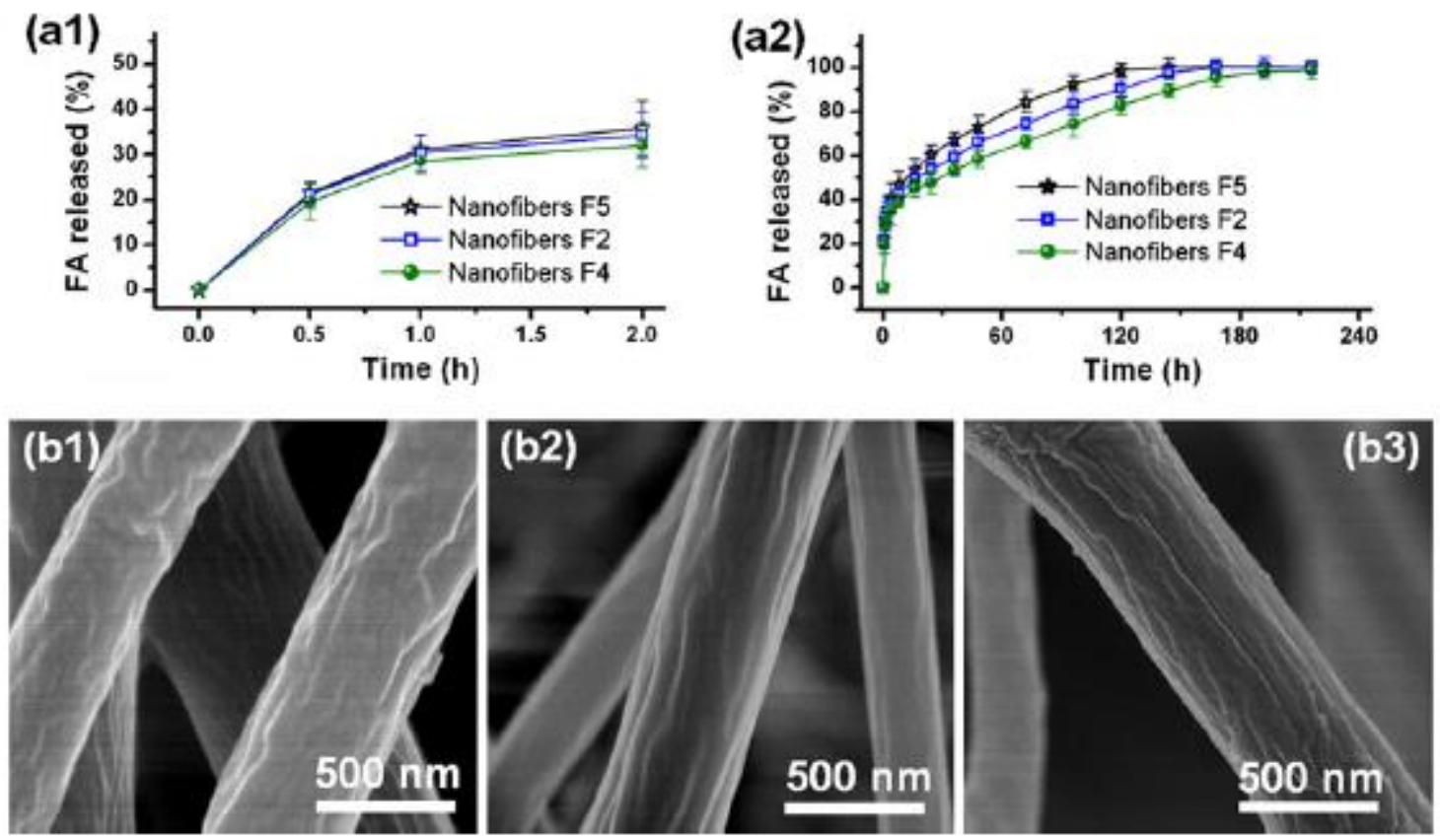

Fig. 9 In vitro drug release profiles: (a1) the initial release and (a2) full-time release, and FESEM images of the final drug-exhausted nanofibers: (b1) F4, (b2) F2, and (b3) F5. 


